\title{
Ромашки спрятались, поникли лютики : analyse des structures Sujet - Verbe /Verbe - Sujet en russe contemporain
}

\author{
Natalia BERNITSKAIIA (Université Paris Sorbonne) \\ Robert ROUDET (Université Lyon 3)
}

\section{Introduction}

Le but de ces lignes est d'analyser les liens sémantico-syntaxiques entre deux propositions juxtaposées du type Sujet - Verbe / Verbe - Sujet (notées $\mathrm{S}_{1} \mathrm{~V}_{1}-\mathrm{S}_{2} \mathrm{~V}_{2}$ : Ромашки спрятались, поникли лютики). Nous comparerons cette structure aux autres structures bipartites analogues, notamment au schéma $\mathrm{S}_{1} \mathrm{~V}_{1}-\mathrm{S}_{2} \mathrm{~V}_{2}$ (Ромашки спрятались, лютики поникли), en essayant d'expliquer les effets sémantiques créés par les différents ordres possibles entre le verbe et le sujet. Notre analyse se limitera aux énoncés dans lesquels les sujets formels, exprimés par les substantifs, sont distincts, et les prédicats sont des verbes perfectifs au passé. (Précisons également que, sur le plan communicatif, tous les énoncés $V_{2} S_{2}$ dont nous traitons, constituent un bloc rhématique dans lequel il est impossible de ménager une pose entre $V_{2}$ et $S_{2}$; cette soudure exclut toute focalisation de l'un des deux constituants). La majorité de nos exemples viennent du corpus Ruscorpora, à l'exception de quelques exemples tirés de nouvelles de Šukšin.

Même s'il existe un assez grand nombre de travaux linguistiques consacrés à l'ordre des mots en russe (cf. par exemple notre bibliographie qui est loin d'être exhaustive), à notre connaissance, la représentation des événements et leur organisation sur la ligne du temps n'ont pas été étudiées en lien avec l'ordre des prédicats. L'étude de l'ordre des mots se limite en général au cadre de la proposition simple. Nous nous proposons donc d'apporter une contribution à ce domaine, non étudié jusqu'ici.

L'hypothèse principale que nous défendons est la suivante : l'inversion de l'ordre "direct » Sujet - Verbe n'est pas, dans une structure $S_{1} V_{1}-S_{2} V_{2}$, comme on le dit souvent dans des termes vagues, un procédé "expressif», "stylistique", mais souvent une nécessité sémantique et syntaxique. Cette hypothèse s'appuie sur les observations suivantes. D'une part, les énoncés VS ne peuvent pas être normalement à l'initiale d'une séquence narrative ; si on peut imaginer qu'un texte commence malgré tout par une affirmation telle que Раздался выстрел ou Вбежала девочка, il est clair que ceci sera un artifice d'écriture. Ce qui est à considérer comme l'amorce normale d'une séquence narrative est une suite "références spatio-temporelles + VS» (Par exemple : На скамейке сидела девочка) ou, de façon sans doute moins fréquente, "références spatio-temporelles + SV (par exemple: В институте Саша учился неважно). II convient toutefois de préciser que certains énoncés VS peuvent, de par leur sémantique même, représenter des références temporelles et pourront par conséquent constituer une initiale, nous avons en vue ici des énoncés tels que Пришла весна.

D'autre part, à l'intérieur d'un texte, alors que les énoncés SV peuvent satisfaire à la nécessité de cohérence textuelle par le fait que $S$, normalement thématique, représente l'élément de liaison avec le contexte de gauche, il n'y a rien de tel dans les énoncés entièrement rhématiques VS. Nous posons donc que lorsqu'il n'y a pas d'éléments thématiques, généralement à sémantique spatio-temporelle, à l'initiale de ces énoncés VS ( $B$ дом вбежала девочка; В лесу раздался выстрел ; На втором этаже открылась дверь), la cohérence textuelle sera assurée par les liens sémantiques existant entre les énoncés $S_{1} V_{1}$ et $V_{2} S_{2}$. Nous tenterons de mettre au jour la façon dont se manifestent ces liens. 


\section{Enoncés événementiels et énoncés catégoriques}

Nous supposons que les énoncés SV et VS différent par leur message communicatif. Pour nous, les énoncés SV sont catégoriques et les énoncés VS sont événementiels ${ }^{1}$.

Les énoncés catégoriques sont les énoncés où il y a un sujet (ce dont on affirme quelque chose) et le prédicat (ce qu'on prédique à propos du sujet). Posant le sujet dans la phrase, on présuppose son existence, donc le prédicat affirme quelque chose à propos d'un objet déjà existant dans la réalité du locuteur ; autrement dit, l'énoncé catégorique Дверь открылась est composé d'une présupposition : il y a une porte (état des choses), et d'une affirmation : cette porte s'ouvrit (prédication à propos de l'état des choses).

Dans les énoncés événementiels, on pose un événement tout entier. Si dans les énoncés catégoriques le locuteur prédique quelque chose à propos du sujet présupposé (autrement dit, le prédicat se réfère au sujet déjà existant dans la réalité linguistique du locuteur), dans les énoncés événementiels, le locuteur prend conscience de l'existence du sujet à travers le prédicat. Ainsi, l'énoncé catégorique Дверь открылась, sera une réponse à la question Что случилось с дверью? alors que l'énoncé Открылась дверь répondra à la question Что случилось? tout court, car il у а d'abord l'événement et c'est cet événement qui fait prendre conscience au locuteur qu'il y a une porte. Cela ne veut pas forcément dire que le locuteur ne voit pas la porte ou qu'il ne connaît pas son existence; cette porte ne fait pas partie des choses construites dans sa conscience linguistique immédiate.

La prise de conscience de l'existence du sujet n'est pas synonyme d'événement inattendu, mais plutôt de passage plus ou moins brusque d'un événement à un autre, un éveil, une soudaine conscience de l'existence d'un phénomène.

II est nécessaire d'établir une classification au sein des énoncés événementiels : le critère en sera la nature de la relation prédicative entre $V$ et $S$ ainsi que leur nature référentielle. Aussi distinguons-nous trois types d'énoncés événementiels :

Type 1 Раздался выстрел. Ici, l'événement est désigné par $S$, tandis que $V$ marque la seule manifestation possible de cet événement. Nous sommes pratiquement dans le cadre de propositions existentielles. La dénomination de l'événement est concentrée dans le constituant nominal, tandis que le verbe est sémantiquement faible. Parmi les verbes figurant dans les énoncés événementiels, on trouve des verbes d'existence (быть, произойти: Произошла катастрофа), des verbes marquant l'apparition, l'émergence (начаться, появиться, прийти: Пришла весна), des manières de se manifester (Пошёл дождь; Раздался выстрел, etc.) [Kovtunova 1976 : 155-156]. Dans ce cas, l'ordre VS est obligatoire en ce sens qu'il n'est pas fonction du contexte mais de la sémantique de $S$ et de $\mathrm{V}$.

Type 2 вбежала девочка. Ici, contrairement au cas précédent, le verbe n'est pas faible sémantiquement; il marque l'apparition, l'émergence et accompagne $S$ qui se réfère à un objet opposable en bloc à d'autres objets de la même classe (Вбежала девочка, собака, учительница...). $P$. Adamec définissait certains de ces énoncés comme marquant ce qu'il nomme "pojavlenie na scene » [Adamec, cité par Kovtunova 1976 : 69]. L'ordre VS est obligatoire, ne dépendant pas du contexte.

Type 3 Открылась дверь. Ici, l'objet auquel renvoie S n'est pas présent dans la conscience linguistique immédiate du locuteur, ce dernier en (re)prend conscience à travers l'événement concernant ce $\mathrm{S}$. A ce stade, l'ordre entre $\mathrm{V}$ et $\mathrm{S}$ dépend du contexte, plus exactement des intentions communicatives du narrateur, il y a donc ici une concurrence entre l'ordre SV et VS.

\footnotetext{
${ }^{1}$ Nous préférons le terme "énoncé événementiel» au terme traditionnel «énoncé thétique ». Comme l'indique André Rousseau, le terme phrase thétique a été introduit par le linguiste pragois Anton Marty (1847 1914) [Rousseau 2008 : 185].
} 


\section{Relations discursives entre SV et VS}

Nous soutenons donc que les événements dénotés par les énoncés SV et VS entretiennent entre eux des relations discursives, c'est-à-dire des relations sémantiques de cohérence, qui motivent l'ordre chronologique des événements : le destinataire doit d'abord découvrir des relations discursives pour déduire, ensuite, une interprétation temporelle. Les relations discursives ont été décrites par différents linguistes et avec différentes terminologies [Akimova, Kozinceva 1987 ; Asher 1993 ; Lascarides \& Ascher 1993; Saussure 2003]. Nous distinguons quatre types de relations discursives au sein de la structure SV-VS : consécution, résultat, explication, caractérisation.

Consécution: Nous entendons par là la consécution simple, c'est-à-dire le cas où l'événement VS est consécutif à l'événement SV mais l'événement VS n'est pas une conséquence causale de l'événement SV. Le lien temporel entre SV et VS est l'antériorité / postériorité. Deux cas sont à distinguer ici : tout d'abord, le repère spatial nécessaire peut être extérieur à l'ensemble SV-VS et être commun à l'ensemble qu'ils constituent; ou bien, il peut être constitué par SV lui-même. Le premier cas est constitué par les énoncés où VS est du type 1 (Раздался выстрел); on aura alors toujours entre $S_{1} V_{1}$ et $V_{2} S_{2}$ un lien abstrait, stéréotypique, c'est-à-dire que les deux événements représentent dans la conscience du locuteur un enchaînement habituel, normal. Les exemples suivants en sont une illustration :

1. Занавес опустился, зажёгся свет. [V. Berežkov Rjadom so Stalinym].

2. Спектакль закончился, раздались аплодисменты. [Exemple construit].

3. Но зритель не пришел, может, передумал идти в кино, а может, сел куда-нибудь на другое место. Свет потух, начался киножурнал. Я снял шапку, вытянул ноги и стал смотреть на экран. [V. Tokareva, Rubl' šest'desjat - ne den'gi].

Dans ces énoncés, un repère spatial, commun aux deux constituants SV et VS, est posé antérieurement dans le contexte de gauche; par exemple, dans les trois cas ci-dessus, ce repère spatial est 8 зале; on peut dire que VS se réfère à ce repère spatial commun à travers SV.

Le second cas, où le repère spatial est donné d'une façon ou d'une autre par $S_{1} V_{1}$, se présente lorsque $\mathrm{V}_{2} \mathrm{~S}_{2}$ est du type 2 (Вбежала девочка), le lien entre SV et VS est souvent uniquement thématique (il n'y a pas de lien conceptuel ou causal) :

4. Дверца открылась, вышел товарищ в сером костюме, обойдя машину, вошел в подъезд. [G. Ja. Baklanov. Žizn', podarennaja dvaždy].

5. Он нажал на клаксон. Дверь дома приоткрылась, выглянула женщина - молодая. Возможно, новая жена деда - я ее еще не видел. [A. Lazarčuk. Vse, sposobnye deržat' oružie].

Dans ces énoncés, SV qui sert de repère spatial pour VS, permet aux verbes de mouvement de VS de ne pas réaliser explicitement leur valence obligatoire, le complément de lieu.

On peut trouver des cas où les deux possibilités envisagées ci-dessus se combinent, car SV représente une référence spatiale, mais un lien conceptuel ou causal existe entre les deux événements :

6. К тому же в этот раз случилось то, что давно должно было произойти: в кастрюльке для чая, поставленной на газ еще до тревоги, выкипела вода, кастрюлька обүглилась, повалил вонючий дым. [D. Rubina. Vo vratax tvoix].

Enfin, la relation de consécution peut se manifester dans les énoncés où VS est du type 3 (Открылась дверь). Dans ces énoncés, la nature rhématique de $S_{2}$ se fait beaucoup moins nette, le lien thématique entre SV et VS se réalise comme une relation de partie / tout : souvent $S_{2}$ représente une partie de $S_{1}$ ou d'un autre objet déjà présent dans le contexte. La présence de $S_{2}$ est déductible à partir des informations données dans le contexte et des connaissances que nous avons sur le 
monde (pour (7), on sait que les ascenseurs ont des portes; pour (8), on sait que le parachute fait partie de l'équipement d'un pilote d'avion) :

7. Лифт остановился, разошлись блестящие двери, и Варвара увидела Ивана. Он полусидел на подоконнике, скрестив в щиколотках босые ноги... [T. Ustinova. Podruga osobogo naznačenija].

8. Летчик нажал на кнопку катапультирования, и его выбросило из падающего самолета вместе с креслом. Потом кресло отделилось, раскрылся парашют, и летчик благополучно приземлился. Это был первый случай катапультирования из реактивного самолета в нашей авиации, и ... [Nauka i žizn' 2006].

On peut remarquer que, dans ces derniers énoncés, l'énoncé VS ne porte pas l'intonation d'achèvement de phrase. L'intonation de non achèvement persistera même s'il y a un point après VS :

9. Паром тем временем подошёл к берегу. Стали въезжать машины. Паромщик орал на шофёров, те бешено крутили рули, то пятились, то двигались вперёд. [Šukšin].

10. Паром подплыл к берегу; стали съезжать машины. Опять гул, рёв, крики... [Šukšin].

Comme nous l'avons remarqué plus haut, les énoncés VS ne peuvent pas, dans des conditions normales, inaugurer une situation nouvelle (sauf le cas particulier des énoncés VS du type Пришла весна); l'événement désigné par l'énoncé VS doit être considéré comme une phase d'un événement plus large. La langue présente la réalité d'une façon subjective ; cette réalité peut être décrite comme un seul événement linguistique ou comme une addition de plusieurs événements (par exemple: Мальчик сунул руку за пазуху и достал оттуда краюху хлеба / Мальчик достал из-за пазухи краюху хлеба). L'énoncé VS fera toujours partie d'une séquence de plusieurs événements décrivant une situation plus globale. Certes, dans une séquence où SV-VS sont liés par une relation de consécution, VS contribue à la progression du récit, mais sans jamais imposer un nouveau décor. En termes de théâtre, on dirait que les énoncés VS ne peuvent pas commencer un nouvel acte de la pièce, ni d'ailleurs en constituer le dénouement final. On pourrait également comparer les énoncés à un train, dont l'énoncé VS ne pourra jamais être la locomotive ni en être la dernière voiture; autrement dit, l'énoncé VS ne peut pas signaler la fin de la situation globale. Le passage à une nouvelle situation se fait selon deux schémas. Dans un premier cas, l'énoncé VS est d'une part le dernier maillon d'un événement plus large, mais il ouvre dans le même temps une perspective narrative ; les événements continuent donc à défiler directement. Dans un deuxième cas, VS impose un arrêt sur l'image (le récit alors stagne), VS est suivi d'une description, d'un commentaire, qui donnent des détails sur cette image et permettent, ensuite, de passer à une nouvelle situation (comme dans les exemples (9) et (10)).

On peut constater sur nos exemples que les structures SV-VS traduisent généralement un rapport de consécution immédiate, l'intervalle entre les deux événements est très court, voire imperceptible. Cependant, on peut trouver des cas dans lesquels l'intervalle entre SV et VS est considérable. Les propositions alors en question sont séparées par un point, et la deuxième proposition $\mathrm{V}_{2} \mathrm{~S}_{2}$ est placée en tête d'un nouvel alinéa. On pourrait croire que ce sont là des signes extérieurs de l'indépendance sémantique des deux propositions, et que la seconde proposition inaugure une nouvelle situation. Ce n'est pas le cas, une proposition VS ne peut pas être indépendante de la situation globale ; cette ponctuation traduit justement un intervalle séparant les événements sans les rendre indépendants l'un de l'autre:

11. Андрей вышел на двор.

Вскоре вышла жена Соня.

- Ох и навезли ! - заговорила она восторженно и с каким-то святым благоговением. [Šukšin].

12. Режиссёр засмеялся.

- Да нет, ничего... Я скоро. - Режиссёр вышел. Пронька закурил.

Вбежала красивая женщина с портфелем. На ходу спросила : 
- Ну, как у вас ? [Šukšin].

Résultat: L'événement décrit par l'énoncé VS est le résultat causé par l'événement donné par $S V$. Comme la relation de consécution, la relation de résultat donne un ordre temporel d'antériorité / postériorité.

Dans ce type d'énoncés, on trouve une relation thématique de partie / tout : $S_{2}$ représente une partie de l'objet global $S_{1}$. VS désigne la phase finale de l'action générale, représentée par I'énoncé SV-VS. L'intervalle entre deux actions est quasi-inexistant, et on est souvent à la limite d'une relation temporelle de simultanéité (recouvrement). On trouve souvent ici la traduction d'un phénomène suivie de l'expression du bruit provoqué par ce premier événement :

13. Постояли Немцы с Григорием у вагона. Состав шевельнулся, заскрипели сцепки. Гриша обнял Олега, потом Люську. [Ju. Družinikov. Viza v pozavčera].

14. Не прошло и нескольких минут, как состав дернулся, загремели сцепки между вагонами, застучали по стыкам рельсов колеса. [R. B. Axmedov. Promel'ki].

15. Дверь захлопнулась, щелкнул замок, и все стихло. [G. M. Markov. Strogovy].

16. Конвоир остановился у железной узкой двери, открыл ее и довольно бесцеремонно втолкнул меня в маленькую камеру со сплошными железными гладкими стенами и небольшой электрической лампой на потолке. Дверь захлопнулась, щелкнул замок. Я остался один. [A. P. Beljaev. Prodavec vozduxa].

17. В этот момент самолет тронулся, заревели моторы, и мы начали выруливать на взлетную полосу. [M. Golovanivskaja. Protivorečie po suti].

18. Лес поредел, но вместо опушки устланная иглами тропа привела меня к воротам. Собственно, это были остатки ворот, каменные столбы, штукатурка осыпалась, обнажилась кирпичная кладка. Дорога со следами колес перешла в липовую аллею. [B. Xazanov. Dalekoe zrelišče lesov].

Explication : L'événement décrit par VS explique (justifie, cause) celui décrit par SV.

Le schéma est ici l'inverse du précédent. (La relation d'explication n'est pas le contraire de la relation de consécution : la relation de consécution peut se fonder uniquement sur un lien thématique, en dehors de tout lien conceptuel ou causal, alors que la relation d'explication exige un lien causal.) La relation temporelle est celle de postériorité / antériorité, comme précédemment à la limite de la simultanéité (recouvrement):

19. Но в 1906 году относительное благополучие семьи кончилось - умер отец. Он укололся иглой, подшивая бумаги, и скончался от заражения крови. [V. Katanjan. Lilja Brik. Žizn'].

20. - Уходит! - крикнул Савелий, продолжая стрелять, но вскоре их автоматы захлебнулись: кончились патроны. - Ушел-таки, сволочь! - в сердцах бросил Савелий и сплюнул песок, набившийся в рот. [V. Docenko. Tridcatogo uničtožit' !].

Caractérisation. La relation de caractérisation comprend de multiples cas de figure. Nous en distinguons trois cas principaux : recouvrement total, recouvrement partiel et encapsulation.

Lorsqu'il s'agit de la relation de recouvrement total, l'événement donné par VS représente une caractéristique, une précision de l'événement général donné par SV ; l'événement VS fait partie de l'événement SV. Comme il ne s'agit pas véritablement de deux événements autonomes, on ne peut parler d'une véritable relation chronologique, mais on peut dire que les limites temporelles pour SV et VS sont les mêmes:

21. Дина улыбнулась, сверкнули зубы. Никоненко показалось, что они бриллиантовые такая была улыбка, - просто, наверное, ему не слишком весело. [T. Ustinova. Bol’šoe zlo $\mathrm{i}$ melkie pakosti].

22. А черт его знает, - Потапов улыбнулся, сверкнули безупречные зубы. [т. Ustinova. Bol'šoe zlo i melkie pakosti]. 
Dans le cas de recouvrement partiel, l'événement décrit par VS coïncide, dans sa phase initiale, avec la phase finale de l'événement décrit par SV. C'est pour cette raison que les verbes figurant dans ces énoncés, sont généralement des antonymes (кончить / начать; прийти / yŭmu). II ne s'agit à nouveau pas ici d'une véritable relation chronologique, le lien temporel entre les deux événements peut être défini comme recouvrement partiel.

On peut dire ici que les propositions SV et VS représentent deux facettes d'une seule et même situation, mais cette représentation se réalise de deux façons différentes: 1) d'une part, l'énoncé SV-VS peut traduire un passage imperceptible d'une situation à l'autre (cf. les exemples(23)(25)) Les deux sujets, de par leur sémantique, désignent des événements par nature immédiatement consécutifs, et la fin du premier correspond donc au début de l'autre; dans ce cas, la situation globale n'est pas exprimée par un troisième énoncé, elle est déduite de la somme des deux propositions SV et VS (en (23), la situation déduite est : le temps a changé, il ne fait plus beau ; en (24) : J'ai changé de vie, de carrière ; en (25) : tout est devenu calme). Dans ce cas, l'événement VS découle de l'événement SV, il y a un lien conceptuel ou causal entre les deux événements :

23. Иван Дмитриевич притянул ее к себе, все втроем они присели над коробкой и смотрели. Жук был квелый, Ванечка успел потерзать его своей любовью. Он лениво шуршал по дну своими мохнатыми лапками, а за окном шелестел дождик. Бабье лето кончилось, наступила осень. [L. Juzefovič. Dom svidanij].

24. Одна моя карьера закончилась, началась другая. Я вспомнил свою первую профессию - сценограф и стал помогать Мюриэль проводить презентации, выставки. [N. Ovčinnikov. Moskva pereživaet stress ot pustoty].

25. Тяжелые шаги богов и их низкие голоса стихли; наступила тишина. [V. Pelevin. Zatvornik i šestipalyj].

2) D'autre part, la situation globale dont les propositions SV et VS sont des facettes, est annoncée explicitement dans le contexte de gauche. Ici, il n'y a pas de lien conceptuel ou causal entre SV et VS :

26. Окружение сменилось раз, потом еще раз. Грузины исчезли, пришли узбеки в тюбетейках. Евреи сменились другими евреями. [S. Jurskij. Ljubimec publiki].

27. Со временем состав учеников значительно изменился: шпана отсеялась, пришли хорошие ребята из новостроечных домов, и школа, дважды сменившая свой номер, стала одной из лучших, если не лучшей в районе. [Ju. Nagibin. T’ma v konce tunnnelja].

Encapsulation : l'événement donné par VS et l'événement donné par SV sont encapsulés dans une situation plus générale qu'ils caractérisent. II s'agit de deux événements autonomes qui coexistent pendant un laps de temps, il est impossible de définir avec exactitude une chronologie entre les événements ; on parle donc d'indétermination temporelle.

Ce type d'énoncés est très fréquent et peut être illustré par les exemples suivants :

28. А еще ему казалось, что он собьется с курса. Но вдруг море успокоилось, появилось солнце, и из воды вышел морской царь Нептун. - Буксирчик, ты отважен и смел, - сказал Нептун. [Malen'kij buksirčik // « Tramvaj » 1991].

29. [...] и то, что он называл своим «я», то тело хотя бы, которое помогло ему совершить все эти пространственные перемещения - теперь всецело находилось во власти Острова. Солнце скрылось, пошел дождь. Тогда он сделал еще один поворот на 90 градусов и прямиком пошел к зданию,[...] [V. Golovanov. Ostrov, ili opravdanie bessyslennyx putešestvij].

C'est ce même type de rapport que traduit ромашки спрятались, поникли лютики, énoncé qui décrit deux facettes d'une même situation (l'automne, la fin de la belle saison) 


\section{Concurrence de l'ordre SV-VS et SV-SV}

Penchons-nous maintenant sur la possibilité des deux ordres $V_{2} S_{2}$ et $S_{2} V_{2}$ dans les cas où ils sont réellement en concurrence. II convient en effet de tenir compte d'un facteur essentiel : dans certains cas, l'ordre $\mathrm{V}_{2} \mathrm{~S}_{2}$ est une contrainte due à la nature même de $\mathrm{V}_{2} \mathrm{~S}_{2}$, dans d'autres cas, plus intéressants pour nous, l'ordre est déterminé par des facteurs contextuels moins évidents. Revenons sur notre hypothèse de départ. On peut supposer que la structure SV-VS correspond à une présentation des deux événements comme formant un tout, un ensemble, et ce pour plusieurs raisons, déjà évoquées, mais que nous voudrions souligner ici. L'une des preuves qu'une structure SV-VS soude les deux événements est l'impossibilité de ces propositions VS d'être à l'initiale d'un texte, comme nous l'avons déjà signalé. Ces propositions sont donc fortement dépendantes du contexte de gauche, elles ne sont pas réellement autonomes. Elles ne représentent qu'un ajout à ce contexte, dans une structure SV-VS la proposition de type VS prend appuis sur la première; ceci nous permet d'affirmer que l'on a une présentation globale de deux événements.

Ainsi, on aura une très nette préférence pour l'ordre VS dans certains cas signalés en particulier dans les relations de caractérisation, où la $2^{\text {ème }}$ proposition ne représente qu'un autre aspect d'un événement en fait unique :

30. Но все на свете течет и меняется: старый босс ушел, пришел новый, нашел, что склад в Манхэттене слишком дорог и слишком мал, приискал новое помещение в настоящем складском районе - настоящий большой склад. [V. Belomlinskaja. Gde paseš' ty ? Gde otdyxaeš' v polden' ]

\section{?? Старый босс ушел, новый пришел}

On peut donc affirmer que la structure SV-VS devient, si ce n'est obligatoire, en tous cas largement préférable, lorsque l'on a un seul et même événement plus ou moins artificiellement scindé en deux. D'autre part, il convient de rappeler que ces énoncés SV-VS ne peuvent que difficilement constituer une fin d'une séquence, ils ouvrent une perspective narrative. Nous voulons dire par là que si un énoncé tel que Лифрт остановился, (и) (наконец) двери открылись peut-être la fin d'une séquence (le fait que les portes s'ouvrent en représente vraiment la fin), un énoncé tel que Лифрт остановился, открылись двери ne le peut normalement pas: on observera d'ailleurs qu'il est fréquent que des structures de ce type soient suivies d'un énoncé supplémentaire introduit par $i$ (dans l'exemple en en question par exemple : Лифт остановился, разошлись блестящие двери, и Варвара увидела Ивана. Ceci se vérifie en (7), (8), (15), (17), (27) et (28))

Du fait que ce schéma SV-VS traduit normalement deux événements si intimement liés qu'ils n'en forment parfois plus qu'un seul, on ne peut pas utiliser ce modèle pour traduire deux phénomènes qui sont naturellement dissociés l'un de l'autre, bien qu'ils puissent former une chaîne causale. Ainsi par exemple la phrase suivante semble tout au moins improbable :

\section{?? Собака заворчала, убежали дети}

Alors qu'un ordre des mots différent rendrait cette phrase tout à fait banale :

Собака заворчала, дети убежали / Собака заворчала, и дети убежали

L'explication de ceci réside dans le fait qu'une structure SV-SV peut traduire tout enchaînement d'événements, que ce soit de simple consécution ou de progression de cause à effet, une structure SV-VS implique la fusion (au moins partielle) de ces deux événements.

Etudions maintenant le cas particulier que représente la variante explicative. L'ordre $V_{2} S_{2}$ peut être ici inversé et, semble-t-il, sans changement de sens notable ; nous pouvons ainsi avoir un énoncé parfaitement acceptable en procédant à cette opération sur la phrase ci-dessus (19) :

Но в 1906 году относительное благополучие семьи кончилось - отец умер.

Nous aurons la même possibilité avec l'exemple (20):

Автоматы захлебнулись: патроны кончились. 
Ici également, pas de changement sémantique notable.

Revenons maintenant sur la phrase que nous avons envisagée plus haut (Собака заворчала, убежали дети) que nous avions donnée pour fort improbable. Cette phrase deviendrait acceptable si l'on considérait que l'on a là une structure explicative : Собака заворчала : убежали дети. Cette explication est certes elle-même étrange, inhabituelle, mais ceci n'est pas un problème linguistique.

\section{Les différentes structures SV-VS et la coordination}

Si on compare les différents groupes SV-VS, on s'aperçoit que certains peuvent être transformés en structure coordonnée par $i$, d'autres ne le peuvent pas (et ceci même lorsque les événements présentés correspondent à deux événements consécutifs, ce qui est l'un des schémas les plus habituels où apparait cette conjonction). Nous constatons ceci en manipulant l'exemple (29) :

Солнце скрылось, пошел дождь. Тогда он сделал еще один поворот на 90 градусов и прямиком пошел к зданию,

Солнще скрылось, и пошел дождь. Тогда он сделал еще один поворот на 90 градусов и прямиком пошел к зданию,

De même:

31. Он нажал на клаксон. Дверь дома приоткрылась, выглянула женщина - молодая. Возможно, новая жена деда - я ее еще не видел. [A. Lazarčuk. Vse, sposobnye deržat' oružie]. Дверь дома приоткрылась, и выглянула женщина

D'autres, au contraire, se prêtent difficilement à cette coordination :

32. Однажды в театре они поднимались вдвоем в лифте. Наверху лифт остановился, открылись двери. На этаже перед лифтом ждали люди. [А. ŠČeglov. F. Ranevskaja : vsja žizn']

?? Однажды в театре они поднимались вдвоем в лифте. Наверху лифт остановился, и открылись двери. На этаже перед лифтом ждали люди.

Cette limitation disparaît dans certaines conditions:

33. Лифт остановился, и двери его разъехались с характерным звуком где-то наверху. [А. Žitkov. Kafedra]

Revenons à ce propos sur les énoncés tels que Дина улыбнулась, сверкнули зубы traduisant des jeux de physionomie. Il est intéressant de comparer les exemples que nous avons cités avec un énoncé SV i SV traduisant le même type de réalité :

34. Лизавета улыбнулась, и белые зубы сверкнули молодо. [G.E. Nikolaeva. Bitva v puti]

La comparaison de ce dernier exemple avec les énoncés précédents appelle quelques commentaires : on constate que non seulement l'ordre des termes a changé ici, mais également qu'une épithète est apparue, et surtout un adverbe (on pourrait faire la même remarque à propos de (33): двери его разъехались с характерным звуком где-то наверху; sans ces éléments nouveaux, ces énoncés seraient assez étranges et même peu acceptables (??Лизавema улыбнулась, и зубы сверкнули). Сесі n'a en fait rien de très étonnant, si l'on considère que cette structure SV-SV correspond à une autonomie plus grande de la deuxième proposition par rapport à la première que ce que l'on observe dans une structure SV-VS ; les différents éléments qui apparaissent dans les derniers exemples ont la même fonction que le changement d'ordre des termes : ils rendent la seconde proposition réellement informative par rapport à la première et lui donne l'autonomie sémantique nécessaire pour que la structure SV-SV soit acceptable. Nous retombons donc sur I'hypothèse formulée ci-dessus selon laquelle la structure SV-VS, lorsqu'elle est en concurrence avec une structure SV-SV, tend à présenter deux événements comme formant un bloc.

Les énoncés du type fin /début, eux non plus, ne donnent pas de résultat satisfaisant si on tente une coordination, comme on le voit sur l'étrangeté de l'exemple (26) remanié : 


\section{?? Окружение сменилось раз, потом еще раз. Грузины исчезли и пришли узбеки в тюбетейках. Евреи сменились другими евреями.}

Ce test de la coordination permet d'avoir un critère formel commode de la fusion plus ou moins grande des deux énoncés.

\section{En guise de conclusion}

Récapitulons en guise de conclusion les différentes observations qui nous permettent de voir dans les énoncés SV-VS une structure plus ou moins soudée :

- $\quad$ Les énoncés VS ne peuvent majoritairement pas représenter un début, ils sont dépendants du contexte de gauche ;

- $\quad$ Sauf cas particulier, il existe un rapport de consécution immédiate (ou de recouvrement) entre les deux événements ;

- $\quad$ Dans un grand nombre de cas, la coordination par $i$, qui marque en quelque sorte que l'on a deux événements distincts, n'est pas possible ou pas souhaitable ;

- Lorsque, de par la nature des procès décrits, on a en fait deux faces d'un même événement, l'ordre SV-VS est de loin préférable; et inversement, lorsque c'est le contraire qui est manifeste (Собака заворчала, дети убежали / Собака заворчала, и дети убежали) la structure SVVS devient inadéquate.

Il convient de noter que seuls les énoncés à valeur explicative sont à part, du fait même de l'inversion de l'ordre chronologique qu'ils impliquent; il ne peut évidemment y être question de rapport de consécution, ni de coordination par $i$. L'inversion de l'ordre VS en SV ne semble guère altérer le sens et il serait par ailleurs imprudent d'affirmer qu'ils représentent une structure soudée.

\section{Bibliographie} Publishers.

Asher, N. (1993), Reference to Abstract Object in Discourse, Dordrecht, Kluwer Academic

Bonnot, Ch. (2012), "Deixis et saillance d'événement : le cas des énoncés à accent non final en russe contemporain", in La saillance, K. Haude et A. Montaut (éds), Faits de langues, n 39, Peter Lang, 2012, pp. 49-63.

Bonnot, Ch. (2006), Lorsque la focalisation porte sur l'ensemble de la relation prédicative : les énoncés à accent non final en russe moderne ", in La focalisation dans les langues, travaux réunis par H. et A. Wlodarczyk, Paris, L'Harmattan, 135-148.

Bonnot, Ch. \& Fougeron, I. (1986), "Intonation de "non-finalité" dans les énoncés coordonnés en russe moderne", in Les particules énonciatives en russe contemporain, Institut d'études slaves et Université Paris VII, Paris, 1986, pp. 66-88.

Breuillard, J. (2008), Questions de linguistique slave. Etudes offertes à Marguerite GuiraudWeber. Publications de l'Université de Provence.

Kovtunova, I. (1976), Sovremennyj russkij jazyk. Porjadok slov i aktual'noe členenie predloženija, Moskva, Prosveščenie.

Lascarides, A. \& Asher, N. (1993), "Temporal Interpretation, Discourse Relations and Commonsense Entailment", Linguistics and Philosophy 16, pp. 437-493.

Mann, W.C. \& Thompson, S.A. (1988), "Rhetorical Structure Theory: toward a Functional Theory of Text Organization", Text 8, pp. 243-281.

Rousseau, A. (2008), "Dualité et ambivalence de la notion de proposition", in Cahiers de I'ILSL, n² 25, 2008, pp. 181-200.

Russkaja Grammatika, 1980, Moskva, Nauka. 
Saussure, L. de (2003), Temps et pertinence. Eléments de pragmatique cognitive du temps, Ed. : De Boeck. Duculot 